\title{
LA ENFERMEDAD EN LA HISTORIOGRAFÍA DE AMÉRICA LATINA MODERNA
}

\author{
Diego Armus
}

Swarthmore College, USA

\section{RESUMEN}

En este artículo se discute las tendencias y tópicos dominantes en la historiografía sobre la enfermedad en América latina moderna. Las tendencias dominantes son la historia sociocultural de la enfermedad, la historia de la salud pública y la nueva historia de la medicina. Los tópicos más trabajados son el estudio de las epidemias, el de la transmisión de saberes desde el centro a las áreas periféricas, la medicina tropical y las dimensiones socioculturales de la enfermedad.

PALABRAS CLAVE: historiografía, enfermedad, salud pública, América latina.

\section{SUMMARY}

This article discusses trends and topics on the current historiography of disease in modern latin America. It identifies three dominant trends: the sociocultural history of disease, the history of public health and the new history of medicine. As for the topics, the most relevant ones area the study of epidemics, the transmission of knowledge from the center to the periphery, tropical medicine, and the sociocultural dimensions of the disease.

KEY WORDS: historiography, public health, latin America.

En las últimas dos décadas el tema de la enfermedad ha comenzado a ganar un lugar destacado en la historiografía latinoamericana ${ }^{1}$. Su crecimiento como subcampo es parte de la actual fragmentación de los estudios históricos — ahora mucho más prolíficos en recortes temáticos que en ambiciosas y abarcativas narrativas- y también de preguntas y enfoques que las ciencias sociales y las humanidades han destacado entre sus preocupaciones.

Esta ostensible presencia de la enfermedad como objeto de reflexión ha sido, y sigue siendo, el resultado de contribuciones originadas en distintas agendas de trabajo.

1 Una versión considerablemente más corta de este artículo aparecerá en Between Malaria and AIDS History and Disease in Modern Latin America (Duke University Press, Diego Armus, editor, 2003). El libro reúne estudios escritos por investigadores latinoamericanos y norteamericanos activos en este subcampo en franco desarrollo y son representativos de las tendencias historiográficas discutidas en estas notas. 
En primer lugar, los esfuerzos por renovar la tradicional historia de la medicina. Luego, la diseminación de modelos interpretativos provenientes de otras disciplinas que por diversas vías encontraron en la enfermedad un nudo problemático. Finalmente, los estudios históricos de la población y de sus condiciones materiales de existencia.

Lo que está surgiendo de este dinámico proceso historiográfico ha sido etiquetado como nueva historia de la medicina, historia de la salud pública, o historia sociocultural de la enfermedad. Tal vez por detrás de cada una de estas etiquetas pueda encontrarse una trama de preocupaciones propias y específicas. Es evidente, sin embargo, que cuando se evalúa lo que estas distintas historias están produciendo, algunos de sus temas - no así, necesariamente, el modo de abordarlos - tienden a repetirse. Es evidente también que todas ellas reconocen que las enfermedades son fenómenos complejos, algo más que un virus o una bacteria. Además de su dimensión biológica, las enfermedades cargan con un repertorio de prácticas y construcciones discursivas que reflejan la historia intelectual e institucional de la medicina, pueden ser una oportunidad para desarrollar y legitimar políticas públicas, canalizar ansiedades sociales de todo tipo, facilitar y justificar el uso de ciertas tecnologías, descubrir aspectos de las identidades individuales y colectivas, sancionar valores culturales y estructurar la interacción entre enfermos y proveedores de atención a la salud. De algún modo, y tal como ha escrito uno de los más influyentes historiadores en este campo, una enfermedad existe luego que se haya llegado a una suerte de acuerdo que da cuenta que se la ha percibido como tal, denominado de un cierto modo y respondido con acciones más o menos específicas ${ }^{2}$. En otras palabras, razones particulares y coyunturas temporales enmarcan la vida y muerte de una enfermedad, su «descubrimiento», ascenso y desaparición.

En estas notas no me propongo hacer un balance exhaustivo y detallado de lo que se ha escrito para el largo período que va desde el último tercio del siglo XIX en adelante $^{3}$. Tampoco me propongo adelantar la agenda de lo que debe hacerse en el futuro, toda vez que entiendo que esas indicaciones pueden ser tan presuntuosas como inútiles. Se trata, solamente, de dar cuenta de la dirección o mejor, las direcciones, que está tomando la historia de la enfermedad. De esa producción historiográfica se desprende que tres han sido y son los tópicos dominantes: la dimensión social y política de las epidemias, las influencias externas en el desarrollo médico-científico y

2 RosenBerg, Ch. (1992), «Framing Disease: Illness, Society, and History», en RosENBERG, Ch. y GoldEn, J. (eds.) Framing disease. Studies in cultural history, New Brunswick, NJ, Rutgers University Press, p. xiii.

3 La cuestión de la enfermedad también ha concitado la atención de estudiosos del período colonial, con algunos trabajos particularmente notables referidos al impacto de la conquista. Para un cuadro bastante actualizado del estado de los conocimientos, véase CoOK, N. D. y LovelL, W. G. (eds.) (1992), The Secret Judgments of God: Native Peoples and Old World Disease in Colonial Spanish America, Norman, University of Oklahoma Press; CoOK, N. D. (1998), Born to Die. Disease and New World Conquest, 14921650, Cambridge, Cambridge University Press. 
en las políticas de salud pública de la región y, finalmente, los usos culturales de la enfermedad.

\section{ESCRIBIENDO LA HISTORIA DE LA ENFERMEDAD: NUEVA HISTORIA DE LA MEDICINA, HISTORIA DE LA SALUD PÚBLICA E HISTORIA SOCIO-CULTURAL DE LA ENFERMEDAD}

Tradicionalmente el tema de la enfermedad ha sido una suerte de coto controlado por los historiadores de la medicina. Fueron ellos los que escribieron no sólo una historia de cambios en los tratamientos sino también las biografías de médicos famosos. Más allá de sus específicos aportes, estas historias parecen haberse empeñado en reconstruir el «inevitable progreso» generado por la medicina diplomada, unificar el pasado de una profesión crecientemente especializada y resaltar cierta ética y filosofía moral que se pretende distintiva, inalterada y emblemática de la práctica médica a lo largo del tiempo. La nueva historia de la medicina, por el contrario, tiende a destacar los inciertos desarrollos del conocimiento médico, dialoga con la historia de la ciencia, discute no sólo el contexto - social, cultural y político - en el cual algunos médicos, instituciones y tratamientos «triunfaron», haciéndose un lugar en la historia, sino también aquellos otros que quedaron perdidos en el olvido. Es una narrativa que se esfuerza por tensionar la historia natural de la enfermedad y algunas dimensiones de su impacto social ${ }^{4}$.

La historia de la salud pública, por su parte, destaca la dimensión política, dirige su mirada al poder, la política, el estado, la profesión médica. Es, en gran medida, una historia atenta a las relaciones entre instituciones de salud con estructuras económicas, sociales y políticas ${ }^{5}$. Es, también, una historia que se piensa útil e instru-

\footnotetext{
4 StePAn, N. (1976), Beginnings of brazilian science. Oswaldo Cruz, medical research and policy, 1890-1920, Nueva York, Science History Publications; CUETO, M. (1989), Excelencia cientifica en la periferia, actividades cientificas e investigación biomédica en el Perú, 1890-1950, Lima, Tarea; BENCHIMOL, J. y TEXEIRA, L. (1993), Cobras, lagartos e outros bichos. Una história comparada dos Institutos Oswaldo Cruz e Butantan, Rio de Janeiro, Editora Ufrj/Casa Oswaldo Cruz; Fernandes, T. (1999), Vacina antivariólica: ciência, técnica e o poder dos homes, 1808-1920, Rio de Janeiro, Editoria Fiocruz; CoutinHo, M. (1999), «Ninety years of Chagas disease: a success story in the periphery», Social Studies of Science, 29, 4, 519-549; QUEVEDO VÉLEZ, E. et al. (1993), «Ciencias médicas, estado y salud en Colombia», Historia Social de de la Ciencia en Colombia, v. 8, Bogotá, Tercer Mundo. DELAPORTE, F. (1995), «Chagas, a lógica e a descoberta», História, Ciências, Saúde-Manguinhos, 1, 39-53.

5 Belmartino, S., Bloch, C., CArnino, M. y Persello, M. (1991), Fundamentos históricos de la construcción de relaciones de poder en el sector salud. Argentina, 1940-1960, Buenos Aires, Ops/Oms; Hochman, G. (1998), A era do saneamento. As bases da política de saúde pública no Brasil, São Paulo, Editora Hucitec/Anpocs; MoRgAN, L. (1993), Community participation in public health: The politics of primary care in Costa Rica, Cambridge, Cambridge University Press; DíAZ-BRIQUETS, S. (1983), The health revolution in Cuba, Austin, The University of Texas Press; HERNÁNDEZ LLAMAS, H. (ed.) (1984), La Atención rural primaria en México, 1930-1980, México D.F., IMSS; HORN, J. (1983) «The mexican revolution and health care, or the health of the mexican revolution», Latin American Perspectives, 10, 4,
} 
mental, toda vez que busca en el pasado lecciones para el presente y el futuro porque asume que la cuestión de la salud es un proceso no cerrado. Así, el pasado debe ser investigado apuntando a facilitar intervenciones que, se supone, pueden incidir - de modo no específico sino general - en la realidad contemporánea, intentando reducir las inevitables incertidumbres que marcan a todo proceso de toma de decisión en materia de salud pública.

Esta mirada, en verdad, retoma el legado de la práctica y los estudios del higienismo de fines del siglo XIX y comienzos del XX y, más tarde, en torno a los años cincuenta, de algunos estudios que ya se presentaban como historias nacionales de la salud pública. Ambos esfuerzos, que reconocían y enfatizaban el carácter social de la enfermedad, son antecedentes relevantes al momento de evaluar la historia de la historiografía sobre la salud en América Latina. Allí están, entonces, los puntos de partida de una serie de trabajos que en algunos casos no harán más que celebrar a los primeros sanitaristas - de modo bastante similar a la tradicional historia de la medicina- $\mathrm{y}$, en otros, se empeñarán en analizar, en clave estructuralista, la cuestión de la salud y la medicina como epifenómenos de las relaciones de producción ${ }^{6}$. Como sea, el énfasis de esta historia de la salud está no tanto en los problemas de la salud individual sino en la de los grupos, en el estudio de las acciones políticas para preservar o restaurar la salud colectiva y en los momentos en que el estado o algunos sectores de la sociedad han impulsado acciones destinadas a combatir una cierta enfermedad a partir de una evaluación que excede lo estrictamente médico y está definitivamente marcada por factores políticos, económicos, culturales, científicos y tecnológicos. Sin duda, en la historia de la salud la medicina pública aparece en clave positiva y progresista, como un feliz resultado de la asociación de la ciencia biomédica con una organización racional de la sociedad donde ciertos profesionales — los médicos sanitaristas en primer lugar - han sabido ofrecer soluciones frente a las enfermedades del mundo moderno. Esta asociación, vista como potencialmente benéfica, fue evaluada a partir de sus logros concretos. Así, el insatisfactorio balance resultante ha sido explicado por algunos, y no sin una gran dosis de esquematismo que prescindía de cualquier matiz nacional o temporal, como un resultado de la condición dependiente de la región ${ }^{7}$. Esta dependencia, se decía, determinaría la existen-

24-39; ABEL, Ch. (1994), Health care in Colombia, c. 1920-c.1950., A preliminary analysis, Londres, Institute of Latin American Studies.

6 Un ejemplo de los enfoques celebratorios en LEONARD, J. (1989), «Carlos Finlay's life and death of yellow jack», Bulletin of the Panamerican Health Organization, 23, 438-452. La interpretación estructuralista en GARCÍA, J. (1981), «La medicina estatal en América Latina (1880-1930),» Revista Latinoamericana de Salud 1, 70-110; y GARCíA, J. (1994), Pensamiento social en salud en América Latina, México DF, Interamericana McGraw Hill/OPS.

7 NAVARRO, V. (1994), «The underdevelopment of health or the health of underdevelopment: An analysis of the distribution of human resources in Latin America», International Journal of Health Services, 4, 1, 5-27. 
cia de una elite dirigente y una estructura de poder económico incapaces o desinteresados en crear y distribuir equitativa y eficientemente recursos y servicios sanitarios. Otros estudios listaban los logros y limitaciones de los proyectos de modernización en materia de salud pública a nivel nacional o para una ciudad en particular, reaccionando contra el esquemático uso del modelo dependentista. Se propusieron mostrar que al menos en ciertos contextos urbanos el balance no ha sido tan negativo y que la condición periférica no fue tan decisiva al momento en que que el estado se lanzó a construir la infraestructura sanitaria básica e intentar reducir las tasas de mortalidad, en particular las ocasionadas por las enfermedades infecciosas ${ }^{8}$.

Comparada con la historia de la medicina y la de la salud pública, la historia sociocultural de la enfermedad es más reciente. Se trata, en verdad, de trabajos de historiadores, demógrafos, sociólogos, antropólogos y críticos culturales que, desde sus propias disciplinas, han descubierto la riqueza, complejidad y posibilidades de la enfermedad y la salud, no sólo como problema sino también como excusa o recurso para discutir otros tópicos. Así, esta historia sociocultural apenas dialoga con la historia de las ciencias biomédicas y se concentra en las dimensiones sociodemográficas de una cierta enfermedad, los procesos de profesionalización y medicalización, las condiciones de vida, los instrumentos e instituciones del control médico y social, el rol del estado en la construcción de la infraestructura sanitaria, las condiciones de trabajo y sus efectos en la mortalidad ${ }^{9}$. En algunos casos, estas historias están fuertemente marcadas por el empirismo y no van más allá de una recolección de datos relevantes para la historia de ciertas enfermedades. En otros el objetivo pareciera

8 ESCUDÉ, C. (1989), «Health in Buenos Aires in the second half of the nineteenth century», en Platt, D. (ed.)., Social welfare, 1850-1950. Australia, Argentina and Canada compared, Londres, The Macmillan Press; ABEL, Ch. (1986) Health, hygiene, and sanitation in Latin America, 1870-1950, Londres, Institute of Latin American Studies, University of London.

9 BLISS, K. (1999), «The science of redemption: Syphilis, sexual promiscuity and reformism in revolutionary Mexico City», Hispanic American Historical Review, 79, 1, 1-40; ARMUS, D. (1984), «Enfermedad, ambiente urbano e higiene social. Rosario entre fines del siglo XIX y comienzos del XX», ARMus, D. (ed.), Sectores populares y vida urbana, Buenos Aires, CLACSO; CARBONETTI, A. (1998), Enfermedad y sociedad. La tuberculosis en la ciudad de Córdoba, 1906-1947, Córdoba, Emecor; MARiÁTEGUi, J. (ed.) (1989), La psiquiatría en América Latina, Buenos Aires, Losada, CAMPOS CoelHo, E. (1999), As profissões imperiais: Medicina, engenharia e advocacia no Rio de Janeiro (1822-1930), Rio de Janeiro, Record; AlvareZ, A. y ReYNoso, D. (1995), Médicos e instituciones de salud. Mar del Plata, 1870-1960, Mar del Plata: HISA/ Universidad Nacional de Mar del Plata; GoNZÁLEZ LEANDRI, R. (1999), Curar, persuadir, gobernar. La construcción histórica de la profesión médica en Buenos Aires, 1852-1886, Madrid, CSIC; CARRILlO, A. (1999), «Nacimiento y muerte de una profesión. Parteras diplomadas en México», Dynamis, 19, 167-190; AGOSTONI, C. (1999), «Sanitation and public works in late nineteenth century Mexico city», Quipu, 12, 2, 187-201; CONTRERA CrUZ, C. (1992), «Ciudad y salud en el Porfiriato: la política urbana y de saneamiento de Puebla, 1880-1906», Siglo XIX, 1,3, 55-76; Chomsky, A. (1996), West indian workers and the United Fruit company in Costa Rica, 1870-1940, Baton Rouge y Londres, Louisiana State University Press; MEADE, T. (1997), 'Civilizing' Rio. Reform and resistance in a brazilian city, 1889-1930, University Park, PA, Penn State Press. 
apuntar a mostrar, sin mayores esfuerzos de problematización, que las condiciones de existencia de los pobres, de los sectores populares, o de los trabajadores han estado, siempre, marcadas por la desdicha o que cualquier iniciativa en materia de salud pública ha sido el resultado de un esfuerzo por aumentar la productividad o garantizar la reproducción de la fuerza de trabajo o que las elites impulsaron las reformas sanitarias por su propia seguridad o que la reforma sanitaria fue el resultado de un arbitrario e inescrupuloso empeño de control liderado por una burocracia profesional ya afirmada en instituciones estatales o, más en general y de modo bastante simplista, que el capitalismo dependiente necesitaba esos cambios ${ }^{10}$. La narrativa socio-cultural de la historia de la enfermedad también se ha apoyado en las interpretaciones foucaltianas de la medicalización y el disciplinamiento. Fueron y siguen siendo una referencia indudablemente inspiradora - especialmente en ciertos círculos intelectuales latinoamericanos, donde hizo impacto antes que entre los grupos latinoamericanistas anglosajones - para trabajos que encontraban en la medicina estatal un arsenal de recursos normalizadores constitutivos de la modernidad. Así, las iniciativas estatales - discursivas o políticas - en materia de salud pública fueron entendidas como esfuerzos de racionalización que, habiendo desarrollado conocimientos y lenguajes disciplinares particulares, estaban destinados a controlar a los individuos y a sus cuerpos $^{11}$. En este contexto las hipotéticas etiologías de ciertas enfermedades terminaban sirviendo, así se ha argumentado, a la manera de instrumentos de regulación social, etiquetamientos de la diferencia y legitimación de sistemas ideológicos y culturales. Estas muy sugerentes referencias teóricas, cuando fueron leídas y aplicadas con rigidez, terminaron obviando el examen de las mediaciones y particularidades que de modo específico - es decir con un tiempo, un lugar y una sociedad históricamente concretos - recorren la trama que tejen el poder, el estado, las políticas

10 PINEO. R., «Public health care in Valparaíso, Chile», en PINEO, R. y BAER, J. (eds.) (1998), Cities of hope: People, protests, and progress in urbanizing Latin America, 1870-1930, Boulder, Co., Westview Press; ReCALDE, H. (1997), La salud de los trabajadores en Buenos Aires (1870-1910) a través de las fuentes médicas (1997), Buenos Aires, Grupo editor universitario; RoSARIO COSTA, N. (1985), Lutas urbanas e controle sanitário. Origens das políticas de saúde no Brasil, Petropolis, Vozes; MURDOCK, C. (1995), «Physicians, the state and public health in Chile, 1881-1891», Journal of Latin American Studies, 27, 551-567.

11 Freire COSTA, J. (1979) Ordem medica e norma familiar, Rio de Janeiro, Edições Graal; LÓPEZ SÁNCHEZ, O. (1998), Enfermas, mentirosas y temperamentales. La concepción médica del cuerpo femenino durante la segunda mitad del siglo XIX, México, Plaza y Valdés; SEVCENKO, N. (1984), A revolta da vacina: mentes insanas em corps rebeldes, São Paulo: Brasiliense; VEZZETTI, H. (1985), «El discurso psiquiátrico», BIAGINI, H. ed., El movimiento positivista argentino, Buenos Aires, Editorial de Belgrano; MARTÍNEZ-VERGNE, T. (1999), Shaping the discourse on space: Charity and its wards in nineteenthcentury San Juan, Puerto Rico. Austin, University of Texas Press; LóPEZ SÁNCHEZ, O. (1998), Enfermas, mentirosas y temperamentales. La concepción médica del curerpo femenino durante la segunda mitad del siglo XIX, México, Plaza y Valdés. 
públicas, los saberes, la vida cotidiana, las percepciones de la enfermedad y las respuestas de la gente común.

En estos tres más o menos renovados abordajes hay un indudable esfuerzo por escapar de las limitaciones y estrecheces que han marcado a la tradicional historia de la medicina. Todos - la nueva historia de la medicina, la historia de la salud pública y la historia sociocultural de la enfermedad - entienden a la medicina como un terreno incierto, donde lo biomédico está penetrado tanto por la subjetividad humana como por los hechos objetivos.Todos ellos, también, se proponen discutir la enfermedad como un problema que además de tener una dimensión biológica se carga de connotaciones sociales, culturales, políticas y económicas. Es cierto, se siguen escribiendo trabajos con énfasis sesgadamente empíricos, foucaltianos, celebratorios, o ignorantes de cualquier tipo de mediaciones entre las acciones médico-sanitarias y los requerimientos del sistema económico. Pero también pareciera estar prefigurándose una narrativa historiográfica interesada en contextualizar e interpretar creativamente la riqueza de las iniciativas originadas en la medicina y la salud pública, no sólo en sus dimensiones disciplinadoras sino también en las humanitarias y asistenciales.

\section{ESCRIBIENDO SOBRE LAS EPIDEMIAS COMO UN PROBLEMA HISTÓRICO}

Fue en torno de las epidemias donde la literatura ha sido más prolífica. Su foco está en los avatares de las enfermedades contagiosas que que azotaron sorpresiva e intensamente las ciudades entre el último tercio del siglo XIX y las primeras décadas del $\mathrm{XX}$ y que, en algunos casos, han vuelto hacerlo en las postrimerías del $\mathrm{XX}^{12}$. Algunas de estas historias enfatizan en las condiciones sociales en que emerge la

12 SCEnNA, M. (1974), Cuando murió Buenos Aires, Buenos Aires, La Bastilla; Oliver, L. (1996) «El cólera y los barrios de Guadalajara»,CUETO, M. (ed.), Salud, cultura y sociedad en América Latina. Nuevas perspectivas históricas, Lima, IEP/OPS; TELAROLLI Jr., R. (1996), Poder e saúde: as epidemias e a formação dos serviços de saúde em São Paulo, São Paulo: UNESP; CANO, B. (1996), «La Influenza española en Tlaxcala (1918)», MALvido, E. y Morales, M. (eds.), Historia de la salud en México, México, NAH; PRIETO, A. (1996) «Rosario: Epidemias, higiene e higienistas en la segunda mitad del siglo XIX», LoBAto, M. (ed.) (1996), Política, médicos y enfermedades. Lecturas de historia de la salud en la Argentina, Buenos Aires, Biblos/Universidad Nacional de Mar del Plata; FLORESCANO, E. y MALVIDO, E. (eds.) (1982), Ensayos sobre la historia de las epidemias en México, México, Instituto Mexicano del Seguro Social; CueTo, M. (1997), El regreso de las epidemias. Salud y sociedad en el Perú del siglo XX, Lima: Instituto de Estudios Peruanos; ARMUS, D. (2000), «El descubrimiento de la enfermedad como problema social», LoBATO, M. (ed.), El Progreso, la modernización y sus límites. Nueva historia argentina, Buenos Aires, Sudamericana; PINEO, R. (1990), «Misery and death in the pearl of the Pacific: Health care in Guayaquil, Ecuador, 1870-1925», Hispanic American Historical Review, 70, 609-638; ADAMO, S. ( 1998), «The sick and the dead: Epidemic and contagious disease in Rio de Janeiro, Brazil», PINEO, R. y BAER, J. (1998); AZEVEDO DE BRITO, N. (1997), «La dansarina: a gripe espanhola e o cotidiano na cidade do Rio de Janeiro», História Ciências Saúde, Manguinhos, IV, 1, 11-30. 
coyuntura epidémica, las técnicas y políticas implementadas para combatirla y las reacciones de los gobiernos, la elite, los grupos profesionales y la gente común. Otras también incluyen un examen detenido de los factores biológicos y ecológicos, articulando un diálogo entre historia social e historia de las ciencias biomédicas. Así, los casos latinoamericanos engrosan una suerte de dramaturgia común a todas las epidemias donde se enlazan los temas del contagio, el temor, la huída, la salvación, la búsqueda de chivos emisarios, los esfuerzos por explicar - cultural, religiosa, o políticamente - la llegada, en un cierto momento, del azote epidémico. Pero esta dramaturgia, es preciso subrayarlo, sólo define los marcos de la experiencia epidémica toda vez que las enfermedades no son iguales, los microorganismos se transmiten y afectan de distinto modo, las estrategias de combate no son las mismas y cada sociedad $-\mathrm{y}$, en ocasiones, sus diversos grupos - pueden dar un sentido específico, particular, a sus consecuencias.

Las epidemias ponen al descubierto el estado de la salud colectiva y la infraestructura sanitaria y de atención. Pueden facilitar iniciativas en materia de salud pública y de ese modo jugar un papel acelerador en la expansión de la autoridad del estado, tanto en el campo de las políticas sociales como en el mundo de la vida privada. Sin embargo, la familiaridad de la sociedad con un cierto mal bien puede preparar el terreno para que se ignore, precisamente porque su persistente presencia lo vacía de algunas de las características asociadas a lo extraordinario y sorpresivo o porque el contexto político — qué intereses pone en juego-, el contexto social —a quiénes afecta - o el contexto geográfico — cuán lejos o cerca está de los centros de poderno lo transforma en una cuestión pública, aún cuando por definición se trate de un problema que afecta de modo masivo a la población.

Antes y después del despegue de la bacteriología moderna las epidemias quedaron estrechamente asociadas al mundo urbano, en particular el de las grandes ciudades y, desde fines del siglo XIX, a la cuestión social. Así, y junto a la creciente aceptación de las explicaciones monocausales de cada mal, las referencias al contexto fueron ineludibles, de la precariedad de los equipamientos colectivos a la vivienda, de la herencia biológica o racial a los hábitos cotidianos de higiene, del ambiente laboral a la alimentación y la pobreza, de la inmigración masiva a las multitudes que se agolpaban, peligrosas, en las ciudades. Con el despuntar del siglo XX la estadística se afirmó como disciplina y en algunos países comenzaron a consolidarse agencias estatales específicamente abocadas a las cuestiones de la salud pública. Y los médicos higienistas primero y los sanitaristas más tarde, casi perfilados como una burocracia especializada, dialogando y compitiendo con otros médicos y otros actores en el ámbito político, religioso o legal, jugarían un rol decisivo en la modernización del equipamiento urbano y las redes de asistencia, reforma y control social.

A veces la lucha antiepidémica desplegaba campañas cuasi militarísticas en su retórica - los microorganismos eran definidos como enemigos - y también en su práctica - alentando intervenciones intrusivas y violentas. Tal vez por eso, en ocasiones, 
fueron resistidas, aun cuando utilizaran recursos que no eran totalmente nuevos para la población. Otras veces, a esas estrategias se sumaban empeños que enfatizaban en la persuasión y la educación, apuntando a difundir entre la población un código higiénico que, en el mediano plazo, logró una tremenda aceptación e impacto en la vida cotidiana.

Sin afectar masivamente a la población algunas enfermedades como la sífilis o la lepra fueron calificadas, en algunos contextos, como epidémicas. Razones sociales, culturales o políticas, legitimadas por el saber médico, las transformaban en problemas nacionales capaces de atraer la atención de la opinión pública y promover campañas específicamente destinadas a erradicarlas. Otras enfermedades, crónicas como la tuberculosis, o las gastrointestinales, o endémicas como la malaria, la anquilostomiasis y la fiebre amarilla, que no irrumpían por sorpresa pero estaban bien instaladas en la trama social y a veces mataban y enfermaban más que las epidémicas, no siempre lograban movilizar recursos materiales, profesionales o simbólicos suficientes como para ser percibidas como serios problemas colectivos. Menos espectaculares, estas enfermedades han hecho un impacto en el mundo urbano o el rural, o en ambos.Y por omnipresentes, menos ruidosas, carentes de terapias específicas exitosas, fuertemente marcadas por las condiciones materiales de existencia o localizadas en los márgenes geográficos o sociales, la gestación de políticas específicas destinadas a combatirlas o no existían o demandaron de ingentes esfuerzos al momento de querer instalar el tema en la opinión pública y en la conciencia de las elites locales y nacionales. Y si en el mundo urbano algunas de estas enfermedades finalmente lograron devenir en asuntos públicos —en gran medida por haber sido percibidas como elementos constitutivos de la cuestión social- en el campo fueron los males endémicos los que facilitaron la ampliación del área de incumbencia de las políticas públicas en materia de salud ${ }^{13}$. En ese contexto, el proyecto de sanear el campo o al menos combatir una de sus endemias reafirmaba el proceso de construcción de la nación y la expansión del estado y del poder central ${ }^{14}$.

13 ARMUS, D. (2000), «Consenso, conflicto y liderazgo en la lucha contra la tuberculosis. Buenos Aires, 1870-1950», en SuRIANO, J. (ed.), La cuestión social en la Argentina, 1870-1943, Buenos Aires, La Colmena; CASTRO SANTOS, L. (1980), «Estado e saúde pública no Brasil (1889-1930)», DadosRevista de Ciências Sociais, 23,2, 237-250; OBREGÓN, D. (1996), «De 'Arbol maldito' a 'Enfermedad curable': los médicos y la construcción de la lepra en Colombia, 1884-1939», en CUETO, M. (ed.) (1996). StePAn, N. (2001), Picturing tropical nature, Ithaca, Nueva York, Cornell University Press; Silva, L. J. da (1999), A evolução da doença de Chagas no estado de São Paulo, São Paulo, Hucitec.

14 CASTRO SANTOS, L. (1993), «A reforma sanitária 'pelo alto': O pionerismo paulista no início de século XX», Dados-Revista de Ciências Sociais, 36, 3, 361-392; FrANCO AGUDELO, S. (1990), El paludismo en América Latina, Guadalajara, Editorial de la Universidad de Guadalajara; HocHMAN, G. (1998). 


\section{ESCRIBIENDO SOBRE LA ENFERMEDAD EN RELACIÓN A LAS INFLUENCIAS EXTERNAS Y LA CONSTRUCCIÓN DE LOS ESTADOS NACIONALES}

Otro tópico relevante ha sido el de la llegada de la medicina europea y norteamericana a América Latina. Se trata, en gran medida, de una reacción contra las interpretaciones difusionistas que asumían una pasiva recepción de conocimientos y prácticas articuladas fuera de la región. Así, el énfasis no está en el transplante e importación de ideas sobre ciertas enfermedades - las llamadas, de modo impreciso, tropicales como la fiebre amarilla, la malaria, la anquilostomiasis - sino en el proceso de selección y ensamblaje, en su creativa reelaboración y modificación de acuerdo a específicos contextos culturales, políticos e institucionales. En ese marco interpretativo, los médicos higienistas y los científicos de la periférica América Latina aparecen como aliados y, en ocasiones, como competidores y cuestionadores de la hegemonía científico/cultural europea o norteamericana. Sus trayectorias los descubren discutiendo entre ellos, animando - antes y después del triunfo de la bacteriología moderna- debates sobre las posibles etiologías de ciertas enfermedades, creando instituciones de excelencia científica, empeñándose en esfuerzos más o menos originales por incidir en las tendencias de la morbilidad y mortalidad $^{15}$.

Inevitablemente esas experiencias e iniciativas necesitaban legitimarse de algún modo $\mathrm{y}$, en ese proceso, quedaban fuertemente asociadas a problemas más vastos como son los de la construcción del estado y la nación, las demandas del capitalismo dependiente, la regeneración y mejoramiento progresivo de la «raza nacional», la reforma social y la renovación de las costumbres. Lo interesante es que las enfermedades que desde finales del siglo XIX permitieron articular estos esfuerzos no han sido necesariamente las mismas en cada país. Así, el cólera, la tuberculosis, la malaria, el mal de Chagas, la sífilis, la lepra y, ya en las postrimerías del siglo XX el SIDA y otra vez el cólera, cargan con una relevancia, una significación simbólica, que sólo

15 BenCHIMOL, J. (1999), Dos micróbios aos mosquitos. Febre amarela e a revolução pasteuriana no Brasil, Río de Janeiro, Editora Fiocruz/Editora UFRJ; CuETO, M. (1996), «Tropical medicine and bacteriology in Boston and Peru: Studies of Carrion's disease in the early twentieth century», Medical History, 40, 344-364; COELHO EDLER, F. (1996), «O debate en torno da medicina experimental no segundo reinado», Historia, Ciência Saúde. Manguinhos, III, 2, 284-299; PEARD, J. (1999), Race, place and medicine. The idea of the tropics in nineteenth-century brazilian medicine, Durham y Londres, Duke University Press; PETRAGIA KROPF, S. et al. (2000), «Doença de Chagas: a construção de um fato científico e de um problema de saúde pública no Brasil», Ciência \& Saúde Coletiva, 5, 2, 347-365; DElaporte, F. (1995); PERLETH, M. (1997), Historical aspects of American Trypanosomiasis (Chagas' Disease) (Frankfurt am Main, Peter Lang); BASTIEN, J. (1998), The kiss of death. Chagas' disease in the Americas (Salt Lake City, The University of Utah Press); DELAPORTE, F. (1999), La maladie de Chagas. Histoire d'un fléau continental, París, Payot; DELAPORTE, F.(1997), "'Romaña's sign'», Journal of the History of Biology, 30, 357-366; COUTINHO, M. (1999), «Ninety years of Chagas Disease: a success story at the periphery», Social Studies of Science, 29, 519-49; SilvA, L. J. da (1999), A evolução da doença de Chagas no estado de São Paulo, São Paulo, Hucitec. 
puede aprehenderse cuando se las contextualiza en la historia nacional, regional o local, cuando se las tensiona con las estructuras demográficas, los niveles de urbanización, los avatares - científicos, tecnológicos, políticos, culturales- que marcan la oferta de estrategias específicas de cura.

En torno de ciertas enfermedades «tropicales» como la malaria, la fiebre amarilla y la anquilostomiasis se articula otro tema conectado a los problemas de la politización de la salud y de la recepción y transferencia de saberes y prácticas. En el centro mismo de estos asuntos está el papel jugado por ciertas agencias internacionales, en particular la Fundación Rockefeller. No hay dudas que sus misiones, presentes entre las décadas del diez y del treinta en casi todos los países de América Latina, son una prueba más del aumento de influencia de los Estados Unidos en la región así como su decisivo rol en la organización de servicios independientes por enfermedad y la promoción, en general, de la medicina curativa y de control técnico de las dolencias en desmedro de una medicina más integral y educativa. Pero el problema es más complejo y, afortunadamente, las visiones maniqueas y simplistas sobre la ingerencia imperialista de la Rockefeller no parecen dominar en la historiografía ${ }^{16}$.

En muchos países de la región la salud como cuestión pública es anterior a la llegada de estas misiones. Durante los dos primeros tercios del siglo XIX dominaron los enfoques miasmáticos y medioambientalistas pero sin producir cambios sanitarios infraestructurales de peso, limitando de ese modo sus efectos en la mortalidad general. Hacia finales del siglo la bacteriología moderna tomará la iniciativa, marcando profundamente la dinámica de muchos de los emprendimientos en materia de salud pública. Fue en ese contexto en que algunas comunidades científicas nacionales tendieron a jerarquizar el estudio de ciertas enfermedades tropicales. Entrenados principalmente en Europa occidental, estos médicos desplegaron novedosos esfuerzos de investigación e intervención antes que sus pares norteamericanos. Sin embargo, la llegada de las misiones Rockefeller fue decisiva en la orientación de las reformas sanitarias, en particular en el mundo rural y respecto de enfermedades que, se creía, podían erradicarse con pocos gastos y en poco tiempo. Más allá de las singularidades y los resultados — desparejos según las países y las enfermedades—, los empeños de la Fundación Rockefeller movilizaron la opinión pública respecto de las condiciones de vida y de salud de los pobres del campo, facilitaron enormemente la centralización de los esfuerzos sanitarios, contribuyeron a consolidar el poder del gobierno central frente a las tradicionales estructuras de poder local y regional y galvanizaron la posición de los Estados Unidos como referencia externa dominante en materia de salud pública. A su modo, animaron un complejo proceso de modernización sanitaria y de distribución

16 Cueto, M. (ed.), (1994), Missionaries of science: The Rockefeller foundation in Latin America, Bloomington, Indiana University Press. 
de sus beneficios que destaca procesos de cooptación, canalización de demandas de la sociedad civil, negociaciones entre sectores técnicos nacionales y foráneos.

La agenda técnico-elitista de las misiones debió lidiar con el desafío de adaptarse a las idiosincracias y percepciones de la enfermedad de la población local, algo que los representantes de la fundación hicieron tan mal o con tanta dificultad como la mayoría de los médicos nativos. En cualquier caso, las relaciones entre médicos nativos y especialistas extranjeros fueron complejas, a veces signadas por la subordinación, la alianza, el pragmatismo, el conflicto o la adaptación de las partes involucradas. Al igual que en el mundo urbano, pero enfrentando otras enfermedades, los problemas de cómo intervenir en el mundo rural, cuán profundo penetrar en sus modos cotidianos, cómo persuadir o cuándo recurrir a la coerción fueron cuestiones ineludibles. Y si en el diseño original estas intervenciones podían reverenciar lo técnico o ser instrumentales en una agenda filantrópica neocolonial, al momento de ser llevadas a la práctica, intencionalmente o no, contribuirían a sentar precedentes y facilitar la construcción de las bases institucionales para futuros desarrollos que, en materia de medicina social y prevención, liderarían actores locales ${ }^{17}$.

\section{ESCRIBIENDO LA HISTORIA SOCIO-CULTURAL DE LA ENFERMEDAD}

El tercer y último tópico que permea a muchas de las nuevas narrativas históricas sobre la enfermedad destaca sus dimensiones culturales y sociales en sentido amplio. Se trata, de una parte, de estudios particularmente interesados en el examen de discursos originados en la medicina. De otra, en los usos metafóricos de la enfermedad. El estimulante y atractivo marco interpretativo foucaltiano motorizó los trabajos sobre la locura y el orden psiquiátrico, sus instituciones específicas, sistemas teóricos y procesos de profesionalización. Así, se ha discutido la locura como un objeto que nace y se transforma en un campo de intersecciones que desbordan los temas propios de la psiquiatría. Cuentan entonces la higiene pública y el espacio manicomial, las utópicas empresas de moralización colectiva, el lugar y rol del orden pisquiátrico en la historia de la construcción del estado ${ }^{18}$. En ese contexto se enfatizó en la emergen-

17 PALMER, S. (1998), «Central american encounters with Rockefeller public health, 1914-1921», GILBERT, J., LEGRAND, C. y SALVATORE, R. (eds.) (1998), Close encounters of empire. Writing the cultural history of U.S.-Latin American relations, Durham y London, Duke University Press; BIRN, A. y SOLÓRZANO, A. (1997), «The hook of hookworm: Public health and the politics of eradication in Mexico», CUNNINGHAM, A. y ANDREWS, B. (eds.), Western medicine as contested knowledge, Manchester, Manchester University Press/St. Martin Press. STEPAN, N. (2001), Picturing tropical nature, Ithaca, Nueva York, Cornell University Press; Silva, L. J. da (1999), A evolução da doença de Chagas no estado de São Paulo, São Paulo, Hucitec.

18 Vezzetti, H. (1983), La locura en la Argentina, Buenos Aires, Folios; GARCíA-HuERTAS, R. (1991), El delincuente y su patología: Medicina, crimen y sociedad en el positivismo argentino, Madrid, Cuadernos Galileo, CSIC. 
cia de un poder médico dedicado a disciplinar los cuerpos, normativizar los umbrales sanitarios generales e influir en las prácticas políticas de la sociedad no tanto como exterioridades sino como inmanencias ${ }^{19}$. El enfoque dominante ahora busca distanciarse de la repetición casi mecánica y simplificadora de los postulados foucaltianos, tratando con mayor o menor éxito de usar de ellos pero de modo matizado y cauteloso, apoyándose en información empírica y explorando tanto los contenidos disciplinadores como los asistenciales de las prácticas psiquiátricas. En algunos casos el énfasis ha ido al examen del lugar de la salud mental en los proceso de modernización, en otros a las instituciones, la consolidación de grupos profesionales o las relaciones de disciplinas como la psicología o el psicoanálisis con la cultura ilustrada y la popular ${ }^{20}$.

En cuanto a los usos metafóricos de la enfermedad algunos estudios han explorado la conexión entre patología y literatura y entre patología, género y cultura popu$\operatorname{lar}^{21}$. Los huidizos y ambiguos significados que recorren los desórdenes físicos y espirituales y los discursos y narraciones que pretenden darle sentido están en el centro de esfuerzos interpretativos muy imaginativos y audaces pero no siempre bien anclados en información empírica ${ }^{22}$.

Los discursos sobre las enfermedades de transmisión sexual, en particular la sífilis y con ella la más vasta problemática de la sexualidad, también fueron discutidos

19 Machado, R., Loureiro, A., LuZ, R. y Muricy, K. (1978), Danação da norma: Medicina social e constituição da psiquiatria no Brasil, Rio de Janeiro, Graal.

20 Ruiz Zevallos, A. (1994), Psiquiatras y locos: Entre la modernización contra los Andes y el nuevo Proyecto de modernidad, Lima, Instituto Pasado y Presente; VezeTti, Hugo, (1996), Aventuras de Freud en el país de los argentinos. De José Ingenieros a Enrique Pichón Rivière, Buenos Aires, Paidós, BALÁN, J. (1991), Cuéntame tu vida: una biografía colectiva del psicoanálisis argentino, Buenos Aires: Planeta; PlotKIN, M. (1999), «Tell me your dreams: Psychoanalysis and popular culture in Buenos Aires, 1930-1950», The Americas, 55, 4, 601-629; AGUEROS, N. y ERASO, Y. (2000), «Saber psiquiátrico e institución manicomial. Hacia una comprensión de las estrategias de 'moralización' en el Asilo Colonia de Oliva (Córdoba, 1914-1934)», Cuadernos de Historia (Córdoba) 2, 7-26; CARRARA, S. (1998), Crime e loucura: O aparecimento do manicômio judiciário na passagem do século, Rio de Janeiro/São Paulo: Eduerj/Edusp; CARrara, S. (1998), Crime e loucura: o aparecimento do manicômio judiciário na passagem do século, Rio de Janeiro/ São Paulo: Eduerj/Edusp. RIVERA GARZA, C., «La vida en reclusión; cotidianidad y Estado en el Manicomio General La Castañeda (México), 1910-1930)», en Armus, D. (ed.) (2002), Entre Médicos y Curanderos. Cultura, Historia y Enfermedad en América Latina Moderna, Buenos Aires, Norma.

21 NouzeIlles, G. (2000), Ficciones somáticas. Naturalismo, nacionalismo y políticas del cuerpo (Argentina 1880-1910), Rosario: Beatriz Viterbo; ARMUS, D. (2000), «El Viaje al Centro. Tísicas, Costureritas y Milonguitas en Buenos Aires, 1900-1930», Boletín del Instituto de Historia Argentina y Americana 22 , segundo semestre.

22 Trigo, B. (2000), Subjects of crisis. Race and gender as disease in Latin America, Hannover: Wesleyan University Press; Nouzeilles, G. (1999), «Políticas médicas de la histeria: Mujeres, salud y representación en el Buenos Aires del fin de siglo», Mora. Revista del Instituto Interdisciplinario de Estudios de Género, 5, 97-112; PORTO, A (1999), «A vida inteira que podia ter sido e que não foi: trajetória do um poeta tísico», História, Ciencias, Saúde-Manguinhos, VI: 523-550. ARMUS, D., «El viaje al centro: tísicas, costureritas y milonguitas en Buenos Aires (1910-1940)», en Armus, D. (2002). 
desde perspectivas fuertemente foucaltianas. Así, la lucha contra las enfermedades venéreas aparece como un recurso para construir implícita o explícitamente una población más permeable a los intereses de una cierta biopolítica que postula frente a los imperativos del sexo el autocontrol y la asunción racional y consciente de las responsabilidades biológicas. Enfocada de este modo, la historia de la sífilis - y también la de la locura - se recortan como capítulos del proceso civilizador de occidente que, en el peor de los casos, terminan disolviendo o ignorando cualquier especificidad regional o nacional. Cuando sí toman registro de ellas, se trata de historias interesadas en conectar la enfermedad con cuestiones como la degeneración de la especie, la raza, la inmigración, la identidad nacional, la esfera pública y la privada ${ }^{23}$. En ese contexto algunos trabajos analizaron la generación de modelos médicos de exclusión - que definen estereotipos, estigmatizan y patologizan comportamientosrespecto de la sexualidad y condición de la mujer, de la homosexualidad y de ciertos grupos inmigratorios y raciales ${ }^{24}$. Otros estudios, en particular los enfocados en el SIDA, discuten la compleja y porosa frontera entre lo privado y lo público en cuestiones de políticas de salud. En ese territorio - pertinente, por otra parte, a la historia de tantas otras enfermedades, en el pasado y en la actualidad - toma forma el problema de la formación histórica de los derechos a la salud y de sus componentes individuales y sociales. Así, mientras algunos encuentran en el SIDA el emergente de una crisis en materia de derechos humanos con una dimensión propia de problemas de salud pública, otros ven allí una crisis de salud pública saturada por la problemática de los derechos humanos ${ }^{25}$.

El tema de la creciente presencia del saber y prácticas médicas también ha estimulado historias generales de la medicina o la salud pública. Algunas, en clave foucaltiana, se han propuesto analizar la consolidación del monopolio de curar en la clase médica, los lugares concretos en que se desarrolló el poder médico como poder absoluto - frente al enfermo, las clases populares, la mujer, los adolescentes y los homosexuales - y, finalmente, el rol del saber médico como coedificador de una nueva y moderna sensibilidad ${ }^{26}$. Otras han buscado armar una historia de la salud a partir de un examen de la génesis, desarrollo y crisis del asistencialismo estatal, ofreciendo una narrativa bastante peculiar puesto que si bien presenta al estado como el gran gestor de las desdichas o fortunas de la salud del pueblo no hay, como sí ocurre

23 CARrara, S. (1996), Tributo a vênus. A luta contra a sifilis no Brasil, da passagem do século aos anos 40, Rio de Janeiro, Editoria Fiocruz.

24 SALESSI, J. (1995), Médicos, maleantes y maricas. Higiene, criminología y homosexualidad en la construcción de la nación argentina. Buenos Aires: 1871-1914, Rosario, Beatriz Viterbo Editora; LEINER, M. (1993), Sexual politics in Cuba: Machismo, homosexuality and AIDS, Boulder, CO, Westview.

25 SCHEPER-HugheS, N. (1994), "AIDS and the social body», Social Science and Medicine 39, 7, 991-1003.

26 BARrÁn, J. (1994), Medicina y sociedad en el Uruguay del novecientos, 3 vols., Montevideo, Ediciones de la Banda Oriental. 
en otras historiografías, un deliberado esfuerzo por reconstruir de modo detallado fenómenos vinculados a la profesionalización y emergencia de instituciones de atención $^{27}$. Sin enfocarse en una enfermedad en particular sino en la medicina o la salud en general, estas ambiciosas historias, mejor o peor ancladas en información empírica y, por momentos - inevitablemente - enumerativas, no dominan en la literatura.

Desde hace ya un tiempo el tono lo han estado dando enfoques más acotados en un estilo que, con éxito dispar, parecen haberse propuesto evitar tanto los determinismos foucaltianos, economicistas o de cualquier otro tipo. Uno de ellos, buceando en los discursos sobre la raza, la ciencia, la medicina, la nacionalidad y el futuro, ha sido el de la eugenesia latinoamericana como una eugenesia dominantemente preventiva, como una apuesta neolamarkiana de mejoramiento social bien diferenciada de la eugenesia anglosajona de las esterilizaciones forzadas y masivos exterminios ${ }^{28}$.

Otro enfoque ha centrado en el estudio de la degeneración como tópico relevante en la construcción de la nacionalidad, tanto en los países donde el tema del trópico y la raza aparecían persistentemente asociados como en los que recibieron importantes contingentes inmigratorios y por eso discutieron políticas selectivas de atracción y admisión de extranjeros ${ }^{29}$. Esta problemática, articulada en torno a la preocupación del estado por construir saludables «razas nacionales», también permea muchos de los estudios centrados en discursos y políticas públicas de bienestar. De una parte, se recorta con fuerza la problemática de preservar o mejorar la salud infantil y de la mujer en su condición de madre ${ }^{30}$. De otra, la de la higiene como una ideología que

27 ILlanes, M. (1993), En Nombre del pueblo, del estado y de la ciencia... Historia social de la salud pública. Chile 1880-1973, Santiago de Chile, Colectivo de atención primaria.

28 STEPAN, N. (1991), The hour of eugenics, Race, gender, and nation in Latin America, Ithaca, Cornell University Press; NARANJO OsORIO, C. y GARCíA GONZÁlEZ, A. (1996), Medicina y racismo en Cuba: la ciencia ante la inmigración canaria en el siglo XX, La Laguna, Taller de Historia; STERN, A. (1999), «Responsible mothers and normal children: eugenics and nationalism in post-revolutionary Mexico, 1920-1940», Journal of Historical Sociology, 12, 4: 369-396. LIMA, N. y HocHMAN, G. (1996), «Condenado pela raça, absolvido pela medicina: o Brasil descoberto pelo movimento sanitarista da Primeira República», en CHOR MAIO, M.; VENTURA SANTOS, R. (1996), Raça, ciência e sociedade, Rio de Janeiro, Editora Fiocruz.

29 SCHWARCZ, L. (1993), O espectaculo das raças: Cientistas, instituções e questão racial no Brasil, 1870-1930, São Paulo, Companhia das Letras; NARANJO OSORIO, C. y GARCÍA GONZÁLEZ, A. (1996), Medicina y racismo en Cuba: la ciencia ante la inmigración canaria en el siglo XX, La Laguna, Tenerife,Taller de Historia; CAÑIZARES, J. (1998), «Entre el ocio y la feminización tropical: Ciencia, elites y estado-nación en Latinoamérica. Siglo XIX», Asclepio, L, 2, 11-31; BorgES, D. (1993), "'Puffy, ugly, slothful and inert': Degeneration in brazilian social thought, 1880-1940», Journal of Latin American Studies, 25, 2, 235-256. PEDrAZA GóMEZ, Z. (2002), «La Difusión de una dietética moderna en Colombia. La revista Cromos entre 1940 y 1986», en Armus, D. (2002); PARKER, D. «Civilizando la Ciudad de los Reyes: higiene y vivienda en Lima, 1890-1920», en Armus, D. (2002).

30 BLuM, A. (1998), «Public Welfare and child circulation, Mexico City, 1877-1925» Journal of Family History 23, 3, 240-271; GUY, D. (1998), «The Pan American child congresses, 1916-1942: Pan Americanism, child reform, and the welfare state in Latin America», Journal of Family History 23, 3, 
permite articular en clave técnica preocupaciones políticas y como un valor, una suerte de cultura, que en el mediano plazo logra, al igual que la educación, ser celebrada por las elites y los sectores populares independientemente de las ideologías. Lo que estos estudios indican es que, más allá del significado que cada grupo pudo haberle dado a esa cultura, la higiene individual y colectiva ha devenido en una práctica civilizatoria impuesta, alentada o aceptada tanto por el poder y la cima de la sociedad como por la gente común ${ }^{31}$.

Como ocurre en otras historiografías, las lecturas foucaltianas o post-foucaltianas de la concentración de poder que los médicos logran como resultado del así llamado proceso de medicalización de la sociedad han hecho un impacto en las historias de la prostitución y del alcoholismo en la región. Así, enfermedades venéreas como la sífilis o la gonorrea son tópicos inevitables aunque no centrales en muchas de esas historias enfocadadas, las más de las veces, en analizar los esfuerzos estatales por controlar el contagio de esos males, regular o prohibir el sexo comercial e intentar modelar la sexualidad de las prostitutas ${ }^{32}$. Así también el alcoholismo, en algunos lugares considerado una enfermedad endémica por la medicina diplomada, ha sido discutido no sólo como un ejemplo de las limitaciones de la práctica y saber médicos y de la propia medicalización sino también como un caso donde las dimensiones sociales, cultu-

272-291; RAmírez De ARellano, A. y SeIPP, C. (1983), Colonialism, catholicism, and contraception: A history of birth control in Puerto Rico, Chapel Hill, University of North Carolina Press; NARI, M. (1996), «Las prácticas anticonceptivas, la disminución de la natalidad y el debate médico en la Argentina, 18901940», en LOBATO, M. (ed.) (1996).

31 ARMus, D. (1996), «Salud y anarquía: la tuberculosis en el discurso libertario argentino, 18701940», en LOBATO, M. (ed.) (1996); ARMUS, D. (1995), «O discurso da regeneração. Espaço urbano, utopías e tuberculose na Buenos Aires, 1870-1930», Estudos Históricos, 16, 235-250; ARMUS, D. (1995), «La idea del verde en la ciudad moderna. Buenos Aires, 1870-1940», Entrepasados. Revista de Historia, 10, 9-22; BARANCOS, D. (1996), La escena iluminada. Ciencias para trabajadores, 1890-1930, Buenos Aires, Plus Ultra; CHAlouB, S. (1996), Cidade febril. Cortiços e epidemias na corte imperial, São Paulo, Companhia das Letras; CASTRO SANTOS, L. (1985), «O pensamento sanitarista na primeira republica: Uma ideologia de construção da nacionalidade», Dados-Revista de Ciências Sociais, 28, 2, 193-210; PALMER, D. (1998), «Civilizing the City of Kings: Hygiene and housing in Lima, Peru», en PINEO, R. y BAER, J. (eds.) en «Cities of hope...»; ARMUS, D. y BELMARTINO, S. (2001), «Enfermedades, Médicos y Cultura Higiénica», en CATTARUZzA, A. (ed.) Los Años Veinte. Nueva Historia Argentina, Buenos Aires: Sudamericana.

32 MCCREERY, D. (1986), «'This life of misery and shame': Female prostitution in Guatemala City, 1880-1920», Journal of Latin American Studies, 18, 2, 333-353; ENGEL, M. (1989), Meretrizes e doutores. O saber medico e a prostitução na cidade do Rio de Janeiro, 1840-1890, Rio de Janeiro, Brasiliense; ABreu Esteves, M. (1989), Meninas perdidas: Os populares e o cotidiano do amor no Rio de Janeiro da «Belle Epoque», Rio de Janeiro, Paz e Terra; RAGO, M. (1991), Os prazeres da noite. Prostitução e codigos de sexualidade femenina em São Paulo, 1890-1930, Rio de Janeiro, Paz e Terra; GUY, D. (1991), Sex and danger in Buenos Aires: Prostitution, family and nation in Argentina, Lincoln, University of Nebraska Press; SuÁREZ Findlay, E. (1999), Imposing decency. The politics of sexuality in Puerto Rico, 1870-1920, Durham y Londres, Duke University Press. 
rales, económicas y políticas del problema son más relevantes que las específicamente médicas o psiquiátricas ${ }^{33}$.

Fue en el marco de estos esfuerzos por historiar el proceso de medicalización que se han explorado las respuestas de los sectores populares urbanos frente a las prácticas compulsivas e intrusivas originadas en las iniciativas de salud pública. En el caso de la vacunación antivariólica, algunos quisieron encontrar en esas respuestas populares motivaciones antigubernamentales articuladas como reacciones morales, como evidencias de sectores de la elite manipulando el descontento de las masas, o como resistencias a determinadas políticas sanitarias ${ }^{34}$. Otros analizaron en detalle los avatares de la vacunación antivariólica y las percepciones y tradiciones de ciertos grupos raciales en relación al control de la viruela. Así, lo que estos estudios están revelando es que no sólo las resistencias a ciertas iniciativas en materia de salud pública fueron indicativas de la distancia social, racial, cultural, religiosa y política que separaba a los pobres de los esfuerzos del estado por higienizar el medio urbano sino también que las medidas preventivas de una enfermedad pueden tener distintos significados entre distintos sectores sociales ${ }^{35}$.

En el caso de los enfermos con tuberculosis se ha indicado su capacidad de respuesta tanto en el plano individual como en el colectivo. En el individual, se estudiaron los modos con que los tuberculosos recusaban los estereotipos que sobre ellos circulaban tanto entre grupos de médicos como entre la gente común. En el colectivo, se analizaron instancias en que los enfermos negociaron e incluso desafiaron al poder médico organizando huelgas, presionando a la clase política y usando y siendo usados por diarios, revistas y la radio con el objeto de facilitar su acceso a tratamientos que no tenían el aval del establishment profesional y académico ${ }^{36}$. Enfermos de cáncer también protagonizaron movimientos sociales orientados a tener acceso a drogas que, ellos crerían, eran efectivas ${ }^{37}$.

33 MenÉndez, E. (1990), Morir de alcohol. Saber y hegemonía médica, México, Alianza Editorial Mexicana. Di LISCIA, M.S. (2002), «Viruela, vacunación en indígenas en la pampa argentina del siglo XIX», en Armus, D. (2002).

34 Murilo De CARVAlho, J. (1987), Os bestializados: o Rio de Janeiro e a republica que não foi, São Paulo, Companhia das letras; NEEDELl, J. (1987), "The revolta contra a vacina of 1904: The revolt against 'modernization' in Belle Epoque Rio de Janeiro», Hispanic American Historical Review, 67, 2, 233-269; MEADE, T. (1986), «Civilizing Rio de Janeiro: the public health campaign and the riot of 1904», Journal of Social History, 20, 2, 301-332.

35 Sebe Meihy, J. y Bertolli Filho, C. (1990), «Historia social da saúde. Opinião pública versus poder. A campanha da vacina, 1904», Estudos CEDHAL, 5; CHALHOUB, S. (1996).

36 ARMUS, D. (1999), «De 'médicos dictadores' y 'pacientes sometidos'. Los tuberculosos en acción. Argentina, 1920-1940,» Allpanchis, XXXI, 53, 219-253; BERTOLLI FILHO, C. (1999), «Antropologia da doença e do doente: Percepções e estratégias da vida dos tuberculosos», História Ciências Saúde Manguinhos, VI, 3, 493-522; ARMUS, D. (2001), «Cuando los Enfermos Hacen Huelga. Argentina 19001940», Estudios Sociales. Revista Universitaria Semestral, XI, 20.

37 IPOLA, E. de, «Estrategias de la creencia en situaciones críticas: El cáncer y la crotoxina en Buenos Aires durante la década del ochenta», en Armus, D. (2002). 
También los enfermos de fiebre amarilla, cólera y malaria resistieron medidas de salud pública que ellos evaluaban como inefectivas o contrarias a sus percepciones de la enfermedad resultante de una mezcla de saberes indígenas e hipocráticos ${ }^{38}$. Al final, estos estudios sobre la viruela, la tuberculosis, el cáncer, la malaria, el cólera y la fiebre amarilla parecen estar indicando por lo menos tres asuntos. En primer lugar, la aceptación, resistencia o abierto empeño por acceder a tratamientos y recursos ofrecidos por las intervenciones de salud pública y prácticas médicas de acuerdo a condiciones impregnadas por el contexto local, cultural y específico de cada enfermedad. En segundo lugar, la necesidad de estudiar las intervenciones de salud pública y su receptividad en la población en el corto y largo plazo, prestando atención no sólo a las coyunturas de contestación sino también a su exitosa - y por esa razón menos estudiada y al mismo tiempo más obvia - incorporación en las prácticas de la gente común. Por último, la existencia de un cierto grado de protagonsimo por parte de los enfermos y en ese sentido la necesidad de reconocerlos como sujetos históricos y no meramente como blancos inermes del saber y prácticas médicos. Estos problemas son relevantes porque dan cuenta de la presencia de la cuestión de la enfermedad y la salud en el complejo proceso de ampliación de la ciudadanía social y lo que, de modo impreciso en el entresiglo y mucho más claramente una vez entrado el siglo XX, se dio en llamar en algunos países de la región «derechos a la salud». Pero si el protagonismo de los enfermos no puede ni debe ignorarse, su relevancia y significación deben ser materia de cuidadosa reflexión. Nada indica que durante la primer mitad del siglo XX los temas de la salud, la enfermedad y los equipamientos sanitarios hayan sido centrales en la agenda del movimiento obrero o sostenido motor de movimientos sociales. Sólo cuando la enfermedad se diluye en otros problemas - la larga lucha por la reducción de la jornada laboral, las condiciones ambientales de trabajo y los esfuerzos organizativos de ayuda mutua de origen étnico o laboral - $\mathrm{O}$ cuando una cierta patología está asociada a ciertas ocupaciones - como es el caso de las así llamadas enfermedades profesionales- esa correlación es hasta cierto punto pertinente. Por fuera de estos escenarios el protagonismo limitado pero real de los enfermos, de los que pueden enfermarse o de los que son blancos de las intervenciones de saneamiento no permite concluir en que se trata de influyentes actores en la gestación de políticas de salud. Lo que sí revela, una vez más, es la complejidad de las relaciones entre quienes quieren curar y quienes necesitan curarse y las variadas percepciones y recursos que circulan en torno de una enfermedad y que exceden holgadamente el mundo de la medicina diplomada.

Este mismo interés por la perspectiva de los enfermos y los pacientes jerarquizó el estudio de las percepciones sobre la enfermedad, la salud, el cuerpo y la muerte

38 Cueto, (1997); Goldman, N. (1990), «El levantamiento de montoneras contra «Gringos»y «Masones» en Tucumán, 1887: Tradición oral y cultura popular», Boletín del Instituto de Historia Argentina y Americana Dr. Emilio Ravignani, 2, 47-74. 
entre distintos grupos étnicos, raciales o sociales. Aun cuando muchos de estos estudios se proponen como excursiones al interior de las medicinas folklóricas y alternativas al saber diplomado y oficial, no faltan los que apuntan a señalar que la gente usa - incluso para objetivos que exceden los vinculados al cuidado y la asistenciade diferentes sistemas de atención y de salud. En otras palabras, se constata la coexistencia, y no mutua exclusión, de varios sistemas de salud que, según las circunstancias, aparecen como las referencias de atención dominantes. Este enfoque atento al consumo por parte de la gente común de ofertas de atención provenientes del campo de la medicina diplomada y de la popular ha comenzado a tener un lugar en la historiografía, sea en el caso de profesionales marginados que recurren a la prensa y el apoyo de los enfermos para hacerse de un lugar público que el establishment académico y profesional les está negando, en el caso de charlatanes capaces de usar discrecionalmente posturas, prácticas y terminología propios de la medicina oficial o, finalmente, en el caso de consumir y acceder al saber de los curadores populares ${ }^{39}$.

Otros estudios, alejándose en forma premeditada de una agenda armada en torno de lo culturalmente exótico y folklórico, jerarquizaron el impacto en comunidades rurales o semirurales de las experiencias laborales modernas, el nivel de ingresos y las relaciones de clase como los factores claves en los modos en que la gente común percibe y confronta los problemas de la salud y la enfermedad. Así, la relevancia social de eventos médicos modernos queda enmarcada en un contexto político y económico específico y en una específica coyuntura temporal. Con esa agenda un estudio encontró una fuerte correlación entre pobreza y $\operatorname{SIDA}^{40}$. Y analizando la emergencia de movimientos sociales de las décadas de 1970 y 1980 enfocados en salud ocupacional y salud medioambiental, se ha subrayado el carácter moderno de las percepciones y acción de quienes, prescindiendo de categorías humorales, religio-

39 Armus (1999); CRANDON-MAlamud, L. (1991), From the fat of our souls. Social change, political process, and medical pluralism in Bolivia, Berkeley, University of California Press; ZULAWSKI, A. (2000), «Hygiene and 'the indian problem'. Ethnicity and medicine in Bolivia, 1910-1920», Latin American Research Review, 35, 2, 107-129; Scheper-Hughes, N. (1992), Death without weeping. The violence of everyday life in Brazil, Berkeley, University of California Press; BASTIEN, J. (1992), Drums and stethoscope. Integrating ethnomedicine and biomedicine in Bolivia, Salt Lake City, University of Utah Press; MóDENA, M. E. (1990) Madres, médicos y curanderos. Diferencia cultural e identidad ideológica, Ciesas, México; Beltrão, J. (2000), «A arte de curar dos profissionais de saúde popular em tempo de cólera: Grão-Pará do século XIX», História, Ciências, Saúde. Manguinhos, vol VI, 833-866; LOYOLA, M. (1984), Médicos e curandeiros. Conflito social e saúde, Difel, São Paulo; TEXEIRA WEBER, B. (1999), Medicina, religião, magia e positivismo na república rio-grandense, 1889-1928, Edusc, Bauru, São Paulo; Sowell, D. (2001), The tale of healer Miguel Perdomo Neira. Medicine, ideologies and power in the nineteenth-century Andes, Wilmington, Scholarly Resources; PALMER, S., «La 'voluntad radiante' del Profesor Carbell. Medicina popular y populismo médico en Costa Rica en el decenio de 1930», en Armus, D. (2002); SowEll, D., «Andanzas de un curandero en Colombia: Miguel Perdomo Neira y la 'lucha entre el buen sentido y la ignorancia ciega'», en Armus, D. (2002); MóDENA, M. «Combinar recursos curatives: un pueblo mexicano en las última décadas del siglo XX», en Armus, D. (2002).

40 FARMER, P., «Brujería, política y concepciones sobre el sida en el Haití rural», en Armus, D. (2002). 
sas o propias de la medicina popular, encontraron en la polución industrial el origen de la enfermedad que les aquejaba ${ }^{41}$.

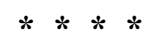

La historia de la enfermedad en la historiografía de la América latina moderna ha crecido de modo desparejo. En Brasil el subcampo existe como tal. Hay revistas académicas, debates, una producción sostenida de tesis de maestría y doctorado sobre temas vinculados a la enfermedad así como centros de estudios, programas de estudios de postgrado, instituciones y archivos que han definido su agenda de trabajo e investigación en torno a las relaciones entre la ciencia, la medicina, la historia y la salud. En el resto de América Latina -incluso entre los países grandes y medianosel balance es muchísimo más modesto y el cuadro que resulta destaca publicaciones peródicas aún en proceso de consolidación, iniciativas individuales, y una suerte de puntillismo que no puede ofrecer más que, en el mejor de los casos, una media docena de trabajos para un cierto tema.

Sin pretensiones de exhaustividad se hizo referencia a legados y contribuciones específicas que son representativos de los tópicos que han animado y animan el crecimiento de la historiografía sobre la enfermedad en la América Latina moderna. Es evidente que sus desarrollos, limitaciones y posibilidades están motorizados por tópicos y tendencias historiográficas que no son exclusivamente latinoamericanos. Así, cualquier intención o tentación de evaluar la historiografía de la enfermedad en la región centrándose única o prioritariamente en las enfermedades tropicales es sesgada y parcial. El exotismo racial, geográfico y cultural de los trópicos ha sido una de las fuerzas que impulsaron el desarrollo de la medicina tropical en los centros académicos imperiales. Su estudio desde las periferias es imprescindible pero en modo alguno suficiente. Incluso en los trópicos otros males han tenido y aún tienen un impacto en la trama social, cultural y demográfica imposible de ignorar.

El cuadro que emerge de esta revisión de los legados y tendencias de la historiografía sobre la enfermedad en América Latina moderna es, en consecuencia, uno que destaca junto a los males del trópico otros asociados a los procesos modernos de urbanización e industrialización. No hay dudas que esta historiografía está tomando nota de la heterogeneidad de la región. Por eso, mientras no olvida la compartida condición neocolonial que ha marcado a todas las experiencias nacionales en los últimos siglo y medio - con múltiples y cambiantes referencias metropolitanasindica, sin ambages, que América Latina es parte de las muchas, en plural, modernidades de occidente.

41 SuSSER, I. (1985), «Union Carbide and the community surrounding it: The case of a community in Puerto Rico», International Journal of Health Services, 15, 4, 561-583. 